\title{
Análisis de los desembarques de la pesca comercial en Yarinacocha (Ucayali, Perú) entre 2015-2019
}

\author{
Analysis of commercial fishing landings in Yarinacocha (Ucayali, Peru) \\ between 2015-2019
}

\author{
Lilia Enny Salazar-Ramírez ${ }^{1,3}$, José Carlos Riofrío-Quijandría², \\ Javier Oscar Zavaleta-Flores ${ }^{1}$, Juan Alfredo Rubio-Rodríguez ${ }^{1}$
}

\section{Resumen}

Se analizaron las estadísticas de desembarque de pescado fresco y refrigerado en Yarinacocha durante el periodo 2015 a 2019, considerando desembarques totales y desembarques por especie (en toneladas métricas - $t$ ), artes de pesca, lugares de pesca y número de viajes. La Captura por Unidad de Esfuerzo (CPUE) se expresó en $t^{*}$ viaje con pesca $^{-1}$. Los resultados indican que los desembarques fueron relativamente mayores en 2016 (815 t), aunque sin diferencias significativas entre años. Los desembarques mensuales presentaron diferencias significativas principalmente entre meses de creciente (enero-abril) con meses de vaciante (julio-agosto), lo que confirma la influencia de los niveles del río, donde los desembarques aumentan en aguas bajas (julio-setiembre) y disminuyen en aguas altas (enero-marzo). En desembarques por categoría trófica, los detritívoros dominaron en «creciente» y los omnívoros en «vaciante». Especies de las órdenes Siluriformes y Characiformes son dominantes, especialmente Pterygoplichthys spp «carachama» y Prochilodus nigricans «boquichico». Las artes de pesca más importantes por su contribución al desembarque fueron las redes de enmalle y las redes de cerco, en tanto que por la frecuencia de uso resaltaron, además, los anzuelos o espineles.

Palabras clave: Amazonia peruana, pesca amazónica, Ucayali, Yarinacocha

\footnotetext{
${ }^{1}$ Área Funcional de Investigaciones de Recursos en Aguas Continentales, Instituto del Mar del Perú. AFIRAC/IMARPE. Callao-Perú

${ }^{2}$ Facultad de Ciencias Biológicas, Universidad Nacional Mayor de San Marcos, Lima, Perú

${ }^{3}$ E-mail: liliasalazarramirez@gmail.com
}

Recibido: 16 de noviembre de 2020

Aceptado para publicación: 12 de junio de 2021

Publicado: 24 de agosto de 2021

CLos autores. Este artículo es publicado por la Rev Inv Vet Perú de la Facultad de Medicina Veterinaria, Universidad Nacional Mayor de San Marcos. Este es un artículo de acceso abierto, distribuido bajo los términos de la licencia Creative Commons Atribución 4.0 Internacional (CC BY 4.0) [https:// creativecommons.org/licenses/by/4.0/deed.es] que permite el uso, distribución y reproducción en cualquier medio, siempre que la obra original sea debidamente citada de su fuente original 
The statistics of landing of fresh and chilled fish in Yarinacocha lake during the period 2015 to 2019 were analysed, considering: total landings and landings by species (in metric tons - $t$ ), fishing gear, fishing locations and number of trips. The catch per unit effort (CPUE) was expressed in $\mathrm{t}^{*}$ fishing trip ${ }^{-1}$. The results indicate that landings were relatively higher in 2016 (815 t), although without significant differences between years. The monthly landings showed significant differences mainly between the months of «high waters» (January-April) with months of «low waters» (July-August), which confirms the influence of river levels, where landings increase in low waters (July-September) and decrease in high waters (January-March). In landings by trophic category, detritivores dominated in «high waters» and omnivores in «low waters». Species of the orders Siluriformes and Characiformes were dominant, especially Pterygoplichthys spp «carachama» and Prochilodus nigricans «boquichico». The most important fishing gears for their contribution to landing were gillnets and purse seines, while hooks also stood out due to the frequency of use.

Keywords: Peruvian Amazon, amazon fishing, Ucayali, Yarinacocha

\section{INTRODUCCIÓN}

Una de las principales actividades en la Amazonia peruana es la pesquería comercial artesanal, la cual genera recursos económicos a la población local. Esta industria realiza los desembarques en las principales ciudades, pero también en los centros poblados ribereños (Hanek, 1982; Tello, 1995; Cadima, 2003, Allan et al., 2005). Esta actividad es multiespecífica por la diversidad de especies explotadas en los ecosistemas acuáticos neotropicales, considerados como megadiversos; sin embargo, solo unas decenas son comercialmente registradas.

La cuenca del Ucayali se considera como uno de los mayores sistemas de producción pesquera, lo que puede atribuirse a una serie de factores como la superficie y edad de la cuenca, condiciones ecológicas y el aporte de nutrientes de la cordillera de los Andes (Lowe-Mc Connell, 1975; Welcomme, 1979; Riofrío, 1998). En la región Ucayali, la laguna de Yarinacocha se encuentra a $7 \mathrm{~km}$ de la capital Pucallpa $\left(8^{\circ} 20203\right.$ LS y $74^{\circ} 342$
03 LO). Tiene su origen en una sección del río Ucayali, que quedó aislada por el desvío de su cauce principal. Su forma es de «J» invertida. En ella se encuentra el desembarcadero de «La Restinga», ubicado en el brazo más corto de la laguna y que por su ubicación geográfica, es uno de los principales lugares de descarga de la pesca obtenida por la flota pesquera comercial al estado frescorefrigerado (Wasiw et al., 2012).

Los desembarques están condicionados por la estacionalidad climática de la región, principalmente la precipitación y los niveles del río Ucayali, distinguiéndose en este último dos importantes periodos contrastantes: la «vaciante» (julio-diciembre) y la «creciente» (enero-junio) (Junk y Furch, 1985). El objetivo de este trabajo fue analizar las estadísticas de pesca registradas por personal del Instituto del Mar del Perú (IMARPE) en la región, determinando cuantitativa $\mathrm{y}$ cualitativamente la producción pesquera desembarcada entre los años 2015 y 2019, con la finalidad de contribuir al conocimiento de la pesquería en Ucayali. 


\section{Materiales y Métodos}

Se utilizó la información obtenida mediante encuestas diarias directas a los pescadores en el desembarcadero de Yarinacocha entre los años 2015 y 2019, como parte del programa de seguimiento de pesquerías amazónicas de IMARPE sobre desembarque total y por especies, artes de pesca empleadas y lugares de pesca frecuentados. Este trabajo se realizó con la finalidad de elaborar el análisis de sus variaciones mensuales, estacionales y anuales y de los aportes de los lugares de pesca, artes y especies de acuerdo con el Desembarque Total Registrado (DTR). Los resultados fueron relacionados con los niveles medios mensuales del rio Ucayali suministrados por la Dirección de Transporte Acuático de Ucayali.

La información diaria se registra entre las 04:00 y 06:00 horas, considerándose el DTR (kg), desembarque por especie (kg), arte de pesca empleado en las capturas y lugares de pesca frecuentados para las capturas. La información procesada se realizó excluyendo la información de los botes «colectivos» por carecer de información del esfuerzo de pesca (representa la unidad de pesca utilizada en un determinado tiempo). La Captura por Unidad de Esfuerzo (CPUE) total y por especie se expresó en $\mathrm{t}{ }_{*}$ viaje con pesca ${ }^{-1}$. Se consideró la capacidad de bodega para estimar el rendimiento de la flota en base a la ecuación: DTR/CB*100, donde DTR $=$ Registro del desembarque en $\mathrm{t}$; $\mathrm{CB}=$ Capacidad de bodega en $t$ ). Los lugares de pesca reportados se agruparon por subcuencas para determinar las más importantes. La lista sistemática de las especies reportadas sigue el orden filogenético establecido en Ortega et al. (2012) y se emplearon los nombres actualizados de las especies reportadas (Jézéquel et al., 2020).

\section{Resultados y Discusión}

\section{Desembarque Pesquero}

Los resultados muestran una relación directa entre el desembarque y el esfuerzo de pesca, presentando valores ligeramente más altos en 2016. No obstante, los desembarques y el rendimiento muestran un descenso entre 2015 hasta 2018 , con una ligera
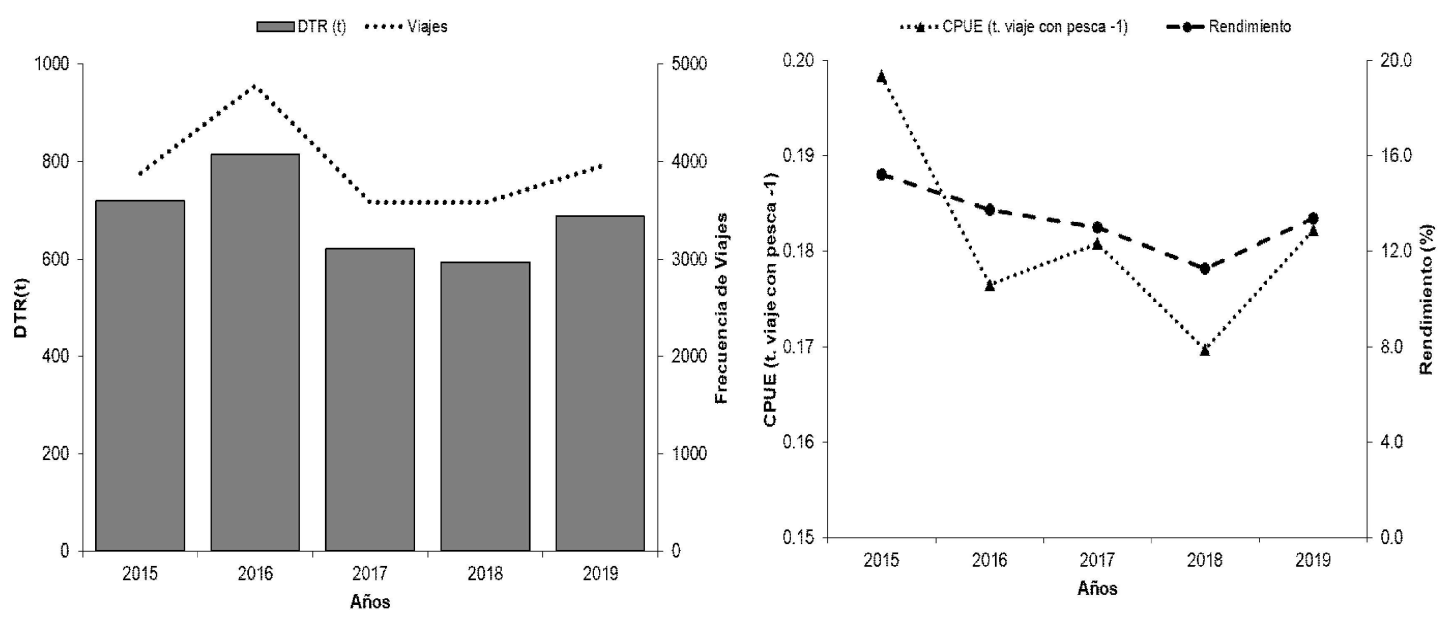

Figura 1. Desembarque total registrado y esfuerzo de pesca (a), captura por unidad de esfuerzo y rendimiento (1) de la flota pesquera comercial de Yarinacocha, Ucayali (2015-2019) 
Cuadro 1. Desembarque de pescado $(\mathrm{t})$ al estado fresco y refrigerado en Yarinacocha (2015 -2019)

\begin{tabular}{lcccccc}
\hline Mes/Año & 2015 & 2016 & 2017 & 2018 & 2019 & Total \\
\hline Enero & 39.4 & 48.9 & 32.7 & 30.2 & 35.1 & 186.3 \\
Febrero & 43.0 & 50.8 & 33.3 & 39.7 & 33.0 & 199.8 \\
Marzo & 56.6 & 46.7 & 38.1 & 31.4 & 31.6 & 204.3 \\
Abril & 7.8 & 49.2 & 43.3 & 41.2 & 52.5 & 193.9 \\
Mayo & 48.3 & 51.4 & 42.5 & 43.2 & 78.0 & 263.5 \\
Junio & 74.1 & 84.3 & 48.7 & 63.5 & 81.7 & 352.4 \\
Julio & 59.3 & 113 & 57.3 & 64.5 & 104.7 & 398.7 \\
Agosto & 71.7 & 60.8 & 77.6 & 71.4 & 87.8 & 369.3 \\
Setiembre & 96.2 & 74.4 & 52.1 & 50.7 & 73.2 & 346.7 \\
Octubre & 107.8 & 82.5 & 71.3 & 60.5 & 37.2 & 359.4 \\
Noviembre & 66.4 & 86.7 & 73.9 & 51.2 & 55.5 & 333.7 \\
Diciembre & 49.2 & 65.7 & 50.8 & 46.3 & 18.5 & 230.5 \\
\hline Total & 719.7 & 814.6 & 621.6 & 593.9 & 688.8 & $3,438.6$ \\
\hline
\end{tabular}

recuperación en 2019, sin diferencias significativas. Los valores de la CPUE no superan los $200 \mathrm{~kg}$ por viaje y las embarcaciones no superan el $16 \%$ de su capacidad de bodega (Figura 1), lo que indica que la actividad de pesca de la flota de Yarinacocha presentaba condiciones de estabilidad relativa en el periodo de análisis con bajos valores de CPUE y de rendimiento.

Según el desembarque mensual (Cuadro 1) y los promedios mensuales multianuales (2015-2019) y contrastado con el promedio mensual entre enero de 2015 y diciembre de 2019 y los niveles medios del rio Ucayali para el mismo periodo (Figura 2), se determina que la mayor producción de pescado se reporta entre junio y noviembre, lo que podría estar asociado con la maduración gonadal y migraciones de reproducción. Asimismo, la mayor producción pesquera se presenta en periodos de «vaciante o estiaje» (Batista y
Petrere, 2003; Barthem y Goulding, 2007; Barletta et al., 2010; Wasiw et al., 2012; García et al., 2012; Vela et al., 2016). La influencia de los pulsos de inundación sobre los mecanismos fisiológicos, ecológicos y etológicos en los recursos pesqueros ha producido una adaptación de la pesquería con el uso de diferentes artes (Cardoso y Carvalho, 2007).

La comparación de los desembarques mensuales mostró que hay diferencias significativas entre los meses de creciente (enero-abril) con meses de vaciante (julio-agosto) (Figura 3), concordando con diversos autores (Batista y Petrere, 2003; Barthem y Goulding, 2007; Barletta et al., 2010; Wasiw et al., 2012; García et al., 2012; Vela et al., 2016), quienes indican que los desembarques de pescado en la Amazonia se encuentran influenciados por los meses de vaciante y creciente. 


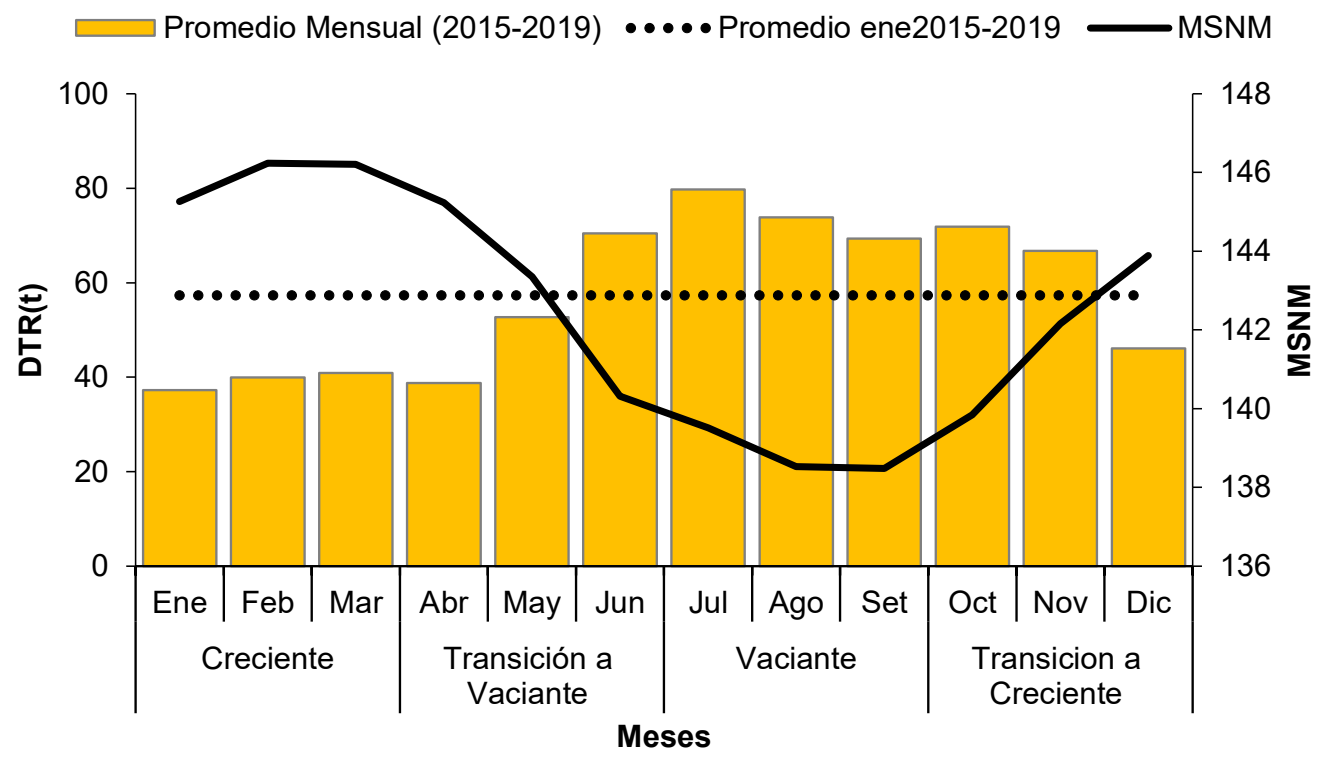

Figura 2. Patrón de desembarque mensual de pescado en Yarinacocha (2015-2019) asociado al nivel medio del rio Ucayali. DTR(t): Desembarque Total Registrado en toneladas; MSNM: metros sobre el nivel del mar

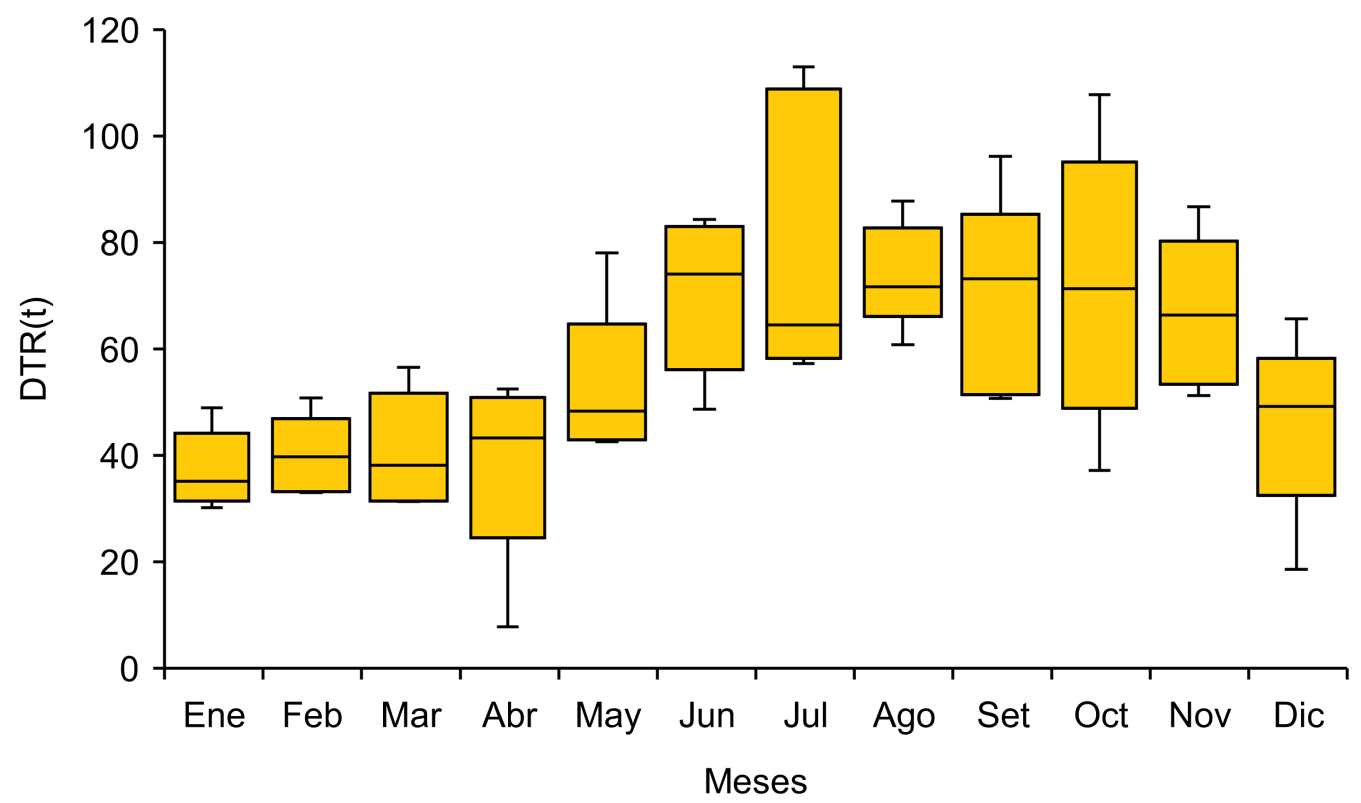

Figura 3. Variación mensual del Desembarque Total Registrado (DTR), en toneladas, de la Flota Pesquera Comercial de Yarinacocha, Ucayali (2015-2019) 


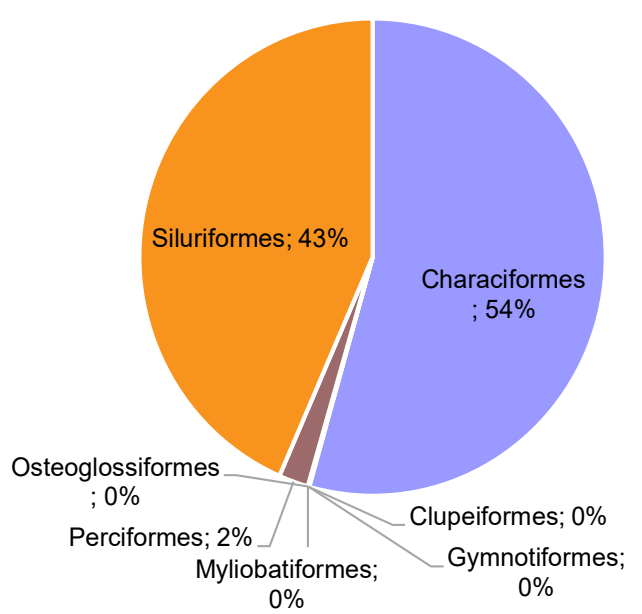

Figura 4. Porcentaje de volumen desembarcado por la Flota Pesquera Comercial de Yarinacocha, Ucayali (2015-2019), según el Orden taxonómico de las especies

\section{Composición Taxonómica de las Espe- cies}

Considerando el Orden de pertenencia de las especies registradas, los Siluriformes cuentan con el mayor número de especies desembarcadas (29), seguido de los Characiformes (25). De acuerdo con el porcentaje del volumen desembarcado, las especies de los principales órdenes fueron Characiformes (54\%) y Siluriformes (43\%). Los otros órdenes tienen aportes inferiores al 10\% del DTR (Figura 4). Es importante anotar que a partir de 2016, los Characiformes incrementan sus aportes al DTR, contrario a la tendencia de los Siluriformes (Figura 5).

De acuerdo con el nivel trófico, los omnívoros son los dominantes, reflejando la importancia de este grupo en el ecosistema como comunidad íctica. El segundo grupo fueron los detritívoros. En este caso, dado que el detritus se puede considerar como no limitante en los ecosistemas amazónicos, se podría soportar una biomasa íctica considerable basada en este tipo de alimentación (Figura 6).
La composición taxonómica de las especies registradas en el desembarcadero de Yarinacocha se muestra en el Cuadro 2. Como se observa, en muchos casos, los nombres comerciales pueden comprender más de una especie; asimismo, una especie puede registrarse con más de un nombre común, concordando con Lopes et al. (2016). No obstante, estos resultados muestran a una pesquería multiespecífica cuyos desembarques totales pueden estar encubriendo la depleción de stocks explotados (Doria et al., 2012). Los resultados indican que en el desembarcadero de Yarinacocha se explotan aproximadamente 63 especies por sus nombres comerciales, que pertenecen a los órdenes Characiformes, Clupeiformes, Gymnotiformes, Myliobatiformes, Osteoglossiformes, Perciformes y Siluriformes, concordando con García et al. (2012), quienes reportan 63 especies en los principales desembarcaderos de la ciudad de Iquitos, Perú. Esta cifra de peces por nombres comerciales involucraría un mayor número de especies comerciales, concordando con Batista y Petrere (2003), quienes reportan 38 grupos de especies de importancia comercial, pero registran 100 especies biológicas.

Durante el periodo hidrológico en «creciente» se determinó que las especies dominantes eran «piro», «carachama», «boquichico» $\mathrm{y}$ «chiochio», con un aporte individual mayor al 10\% del DTR; mientras que, en «transición a vaciante», «boquichico», «palometa», «carachama» y «sardina» fueron las de mayor volumen en los desembarques. De otra parte, las especies que predominaron en vaciante y transición a creciente fueron «carachama» $\mathrm{y}$ «boquichico», con más del $40 \%$ al DTR en conjunto (Figura 7). Acorde con lo mencionado, la «carachama» es la especie con mayor desembarque en todos los periodos hidrológicos, esto es debido a que durante la «vaciante» y transición a «creciente» las zonas de anidamiento de la «carachama» se vuelven disponibles (información proporcionada por pescadores de Yarinacocha). 


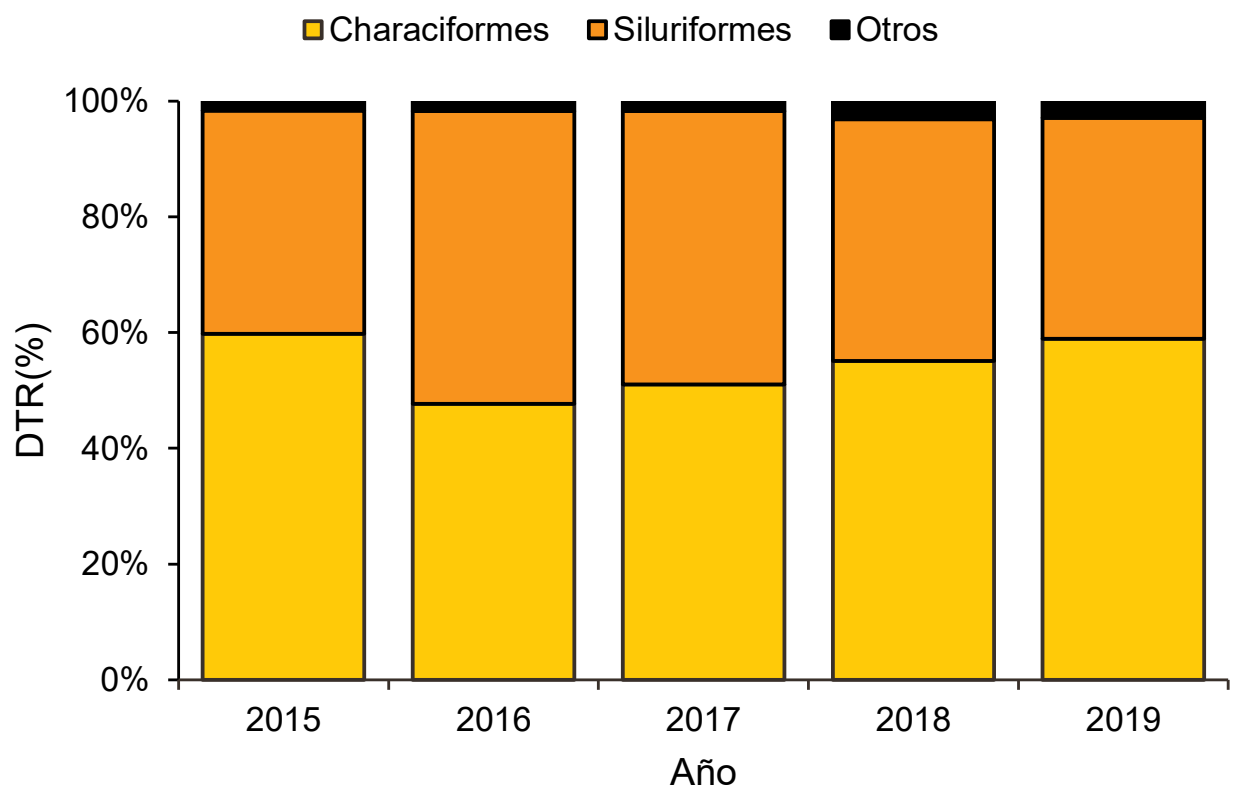

Figura 5. Variación anual (\%) por orden taxonómico de especies desembarcadas por la Flota Pesquera Comercial de Yarinacocha, Ucayali (2015-2019). DTR(t): Desembarque Total Registrado en toneladas

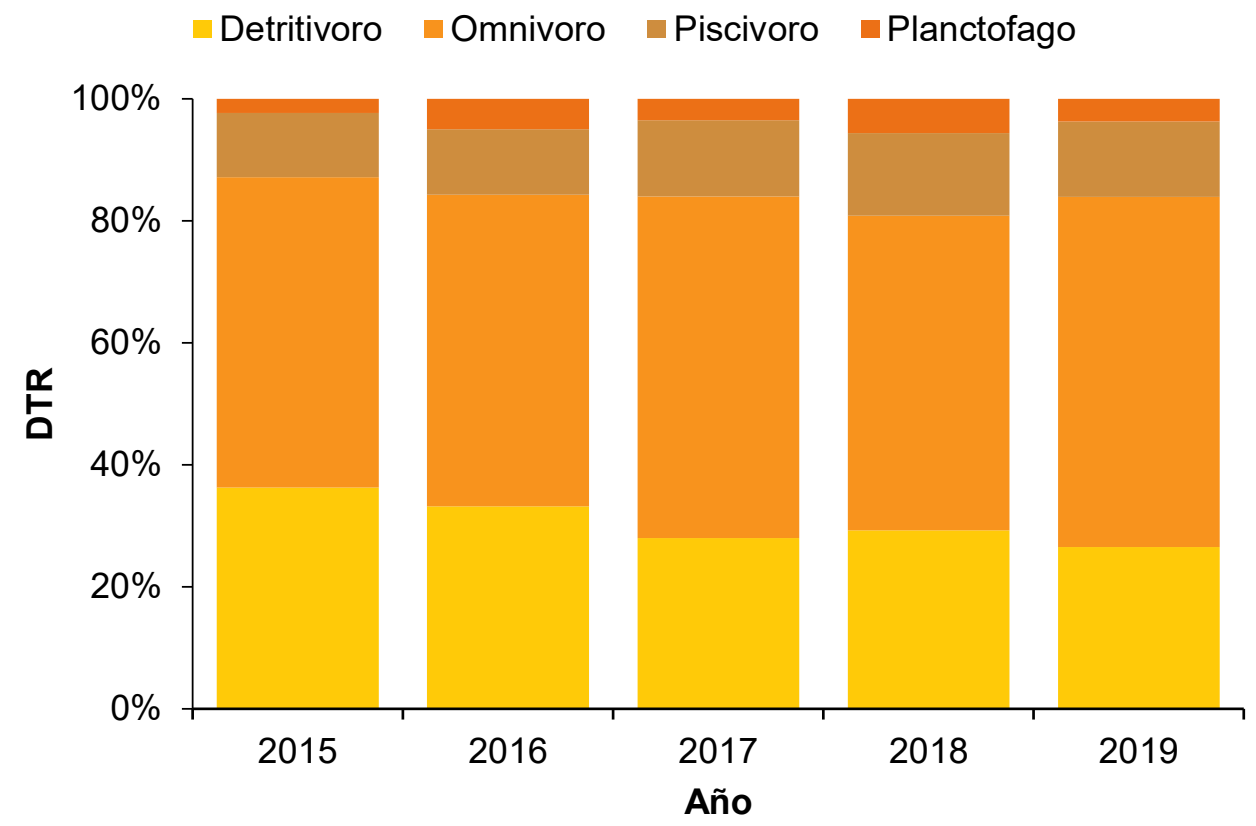

Figura 6. Variación anual (\%) por Nivel Trófico de especies desembarcadas por la Flota Pesquera Comercial de Yarinacocha, Ucayali (2015-2019). DTR(t): Desembarque Total Registrado en toneladas 
Cuadro 2. Lista taxonómica de especies registradas en la pesca comercial de Yarinacocha, Ucayali (Periodo 2015-2019)

\begin{tabular}{|c|c|c|c|}
\hline Orden & Familia & Especie & Nombre Común \\
\hline \multirow[t]{25}{*}{ Characiformes } & Acestrorhynchidae & Acestrorhynchus spp. & Pez Zorro \\
\hline & \multirow[t]{3}{*}{ Anostomidae } & Megaleporinus trifasciatus & Lisa Tres Bandas \\
\hline & & Rhytiodus microlepis & Lisa Negra \\
\hline & & Schizodon fasciatus & Lisa Cuatro Bandas \\
\hline & \multirow[t]{10}{*}{ Characidae } & Brycon amazonicus & Sábalo cola roja \\
\hline & & Colossoma macropomum & Gamitana \\
\hline & & Myloplus rubripinnis & Curuhuara \\
\hline & & Mylossoma albiscopum & Palometa \\
\hline & & Piaractus brachypomus & Paco \\
\hline & & Pygocentrus nattereri & Paña roja \\
\hline & & Roeboides myersii & Dentón \\
\hline & & Tetragonopterus argenteus & Mojara \\
\hline & & Triportheus angulatus & Sardina \\
\hline & & Triportheus auritus & Sardina Macho \\
\hline & \multirow[t]{5}{*}{ Curimatidae } & Curimatella meyeri & San Pedro \\
\hline & & Potamorhina altamazonica & Llambina \\
\hline & & Potamorhina latior & Llambina fila pecho \\
\hline & & Psectrogaster rutiloides & Chiochio \\
\hline & & Psectrogaster amazonica & Ractacara \\
\hline & \multirow[t]{2}{*}{ Cynodontidae } & Cynodon gibbus & Huapeta \\
\hline & & Hydrolycus scomberoides & Chambira \\
\hline & \multirow[t]{2}{*}{ Erythrinidae } & Hoplerythrinus unitaeniatus & Shuyo \\
\hline & & Hoplias malabaricus & Fasaco \\
\hline & Hemiodontidae & Anodus elongatus & Julilla \\
\hline & Prochilodontidae & Prochilodus nigricans & Boquichico \\
\hline \multirow[t]{2}{*}{ Clupeiformes } & Engraulididae & Lycengraulis batessi & Chullacho \\
\hline & Pristigasteridae & Pellona castelnaeana & Panshin \\
\hline Gymnotiformes & Sternopygidae & Sternopygus spp. & Macana \\
\hline Myliobatiformes & Potamotrygonidae & Potamotrygon spp. & Raya \\
\hline \multirow[t]{2}{*}{ Osteoglossiformes } & Arapaimidae & Arapaima gigas & Paiche \\
\hline & Osteoglossidae & Osteoglossum bicirrhosum & Arahuana \\
\hline \multirow[t]{5}{*}{ Perciformes } & \multirow[t]{4}{*}{ Cichlidae } & Astronotus ocellatus & Acarahuazu \\
\hline & & Cichla monoculus & Tucunare \\
\hline & & Cichlasoma amazonarum, Satanoperca spp. & Bujurqui \\
\hline & & Crenicichla spp. & Añashua \\
\hline & Sciaenidae & Plagioscion squamosissimus & Corvina \\
\hline \multirow[t]{27}{*}{ Siluriformes } & \multirow[t]{3}{*}{ Auchenipteridae } & Ageneiosus spp. & Bocon \\
\hline & & Auchenipterus spp. & Novia \\
\hline & & Epapterus dispilurus & Leguia \\
\hline & Callichthyidae & Hoplosternum littorale & Shirui \\
\hline & \multirow[t]{3}{*}{ Doradidae } & Megalodoras irwini & Piro \\
\hline & & Oxydoras niger & Turushuqui \\
\hline & & Pterodoras granulosus & Cahuara \\
\hline & \multirow[t]{2}{*}{ Loricariidae } & Glytopteriichthys spp., Pterygopiichthys spp. & Carachama \\
\hline & & Loricaria spp., Loricariichthys spp. & Shitari \\
\hline & \multirow[t]{18}{*}{ Pimelodidae } & Brachyplatystoma capapretum & Filiote \\
\hline & & Brachyplatystoma filamentosum & Saltón \\
\hline & & Brachyplatystoma juruense & Achune \\
\hline & & Brachyplatystoma rousseauxii & Dorado \\
\hline & & Brachyplatystoma vaillantii & Manitoa \\
\hline & & Calophysus macropterus & Mota \\
\hline & & Brachyplatystoma platynemum & Vaselina \\
\hline & & Hemisorubim platyrhynchos & Toa \\
\hline & & Hypophthalmus edentatus & Maparate \\
\hline & & Hypophthalmus marginatus & Maparate con horquilla \\
\hline & & Leiarius marmoratus & Ashara \\
\hline & & Phractocephalus hemioliopterus & Torre \\
\hline & & Pimelodus blochii & Bagre \\
\hline & & Pseudoplatystoma punctifer & Doncella \\
\hline & & Pseudoplatystoma tigrinum & Tigre Zungaro \\
\hline & & Sorubim lima & Shiripira \\
\hline & & Sorubimichthys planiceps & Achacubo \\
\hline & & Zungaro zungaro & Llausa, Zungaro \\
\hline
\end{tabular}



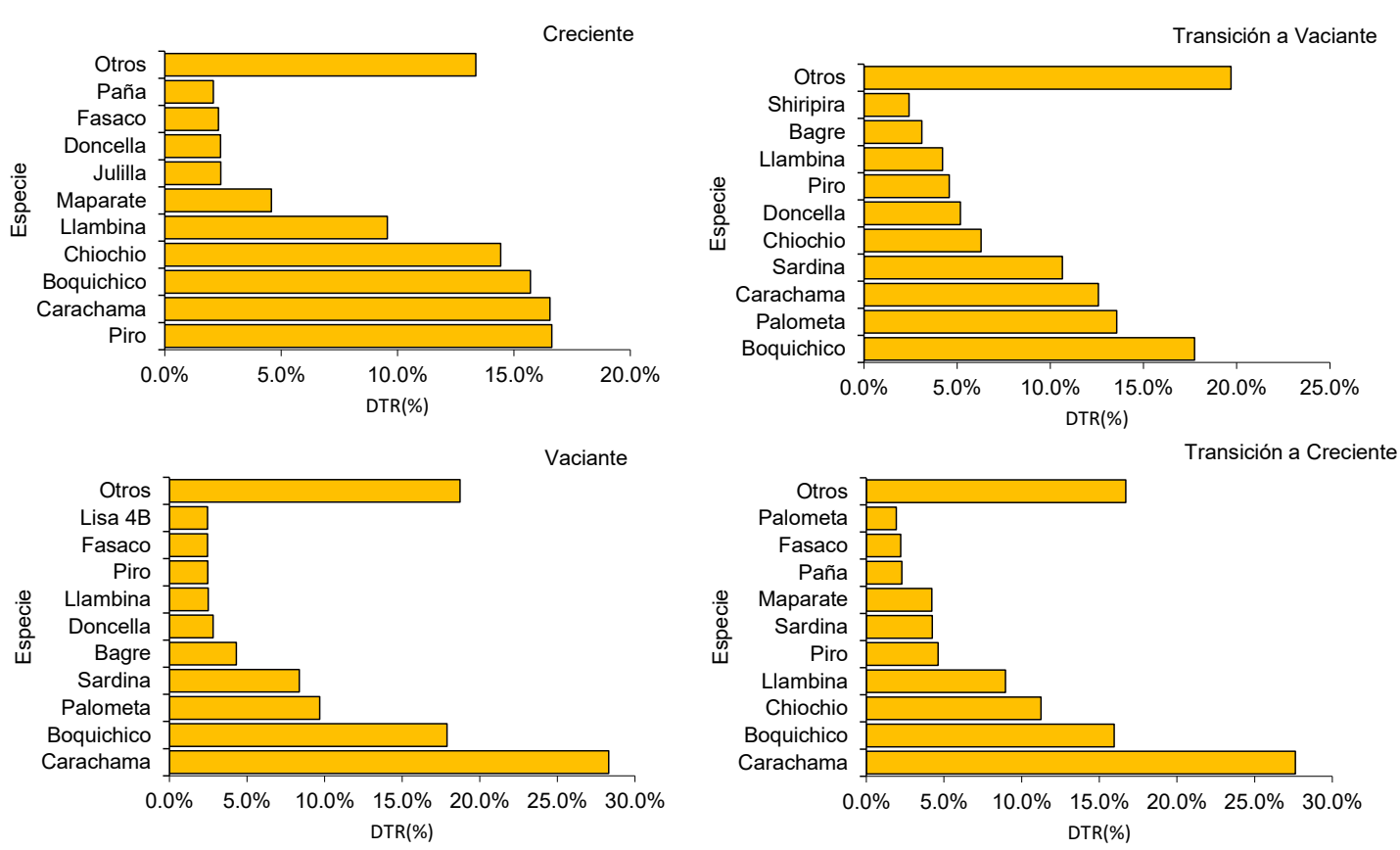

Figura 7. Especies desembarcadas por la Flota Pesquera Comercial de Yarinacocha, Ucayali (2015-2019), según los periodos hidrológicos. DTR(t): Desembarque Total Registrado en toneladas

\section{Artes de Pesca}

Se identificaron los siguientes artes y aparejos de pesca: red de enmalle (regionalmente llamada trampera), red de cerco (hondera), red de arrastre (rastrera), anzuelos-espineles. Entre estos, las redes de enmalle y de cerco aportan aproximadamente el $88 \%$ del DTR (26 y $62 \%$, respectivamente) (Figura 8). En forma similar, Fernandes et al. (2009) indican que el mayor aporte al desembarque en Manaus y Manacajpuru, Brasil, proveniente de pesquerías multi-específicas, se debe al uso de redes de enmalle. No obstante, se reporta para desembarques de pescado fresco en el puerto de Pucallpa, Ucayali, una mayor contribución por las redes de cerco (66\%) en comparación con otras artes (Zorrilla et al., 2016.)

Las redes de enmalle, cerco y anzueloespinel se usan durante todo el año, variando el aporte de las redes de enmalle entre 3 y 68 $\mathrm{t}(34.7 \pm 14.9 \mathrm{t})$, registrándose los valores más altos en 2016. Por otro lado, las redes de cerco presentaron valores de captura media de $14.5 \pm 9.4 \mathrm{t}$, con un valor máximo de $45 \mathrm{t}$

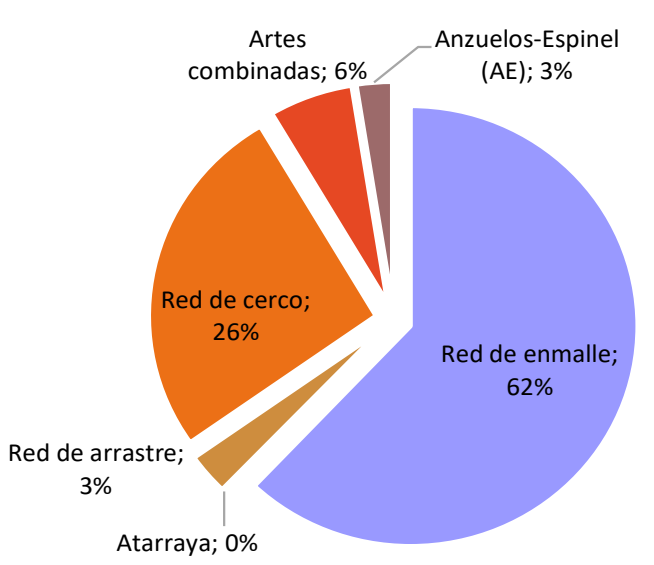

Figura 8. Aporte al Desembarque Total Registrado (DTR, \%) de las artes de pesca de la Flota Pesquera Comercial de Yarinacocha, Ucayali (2015-2019) 


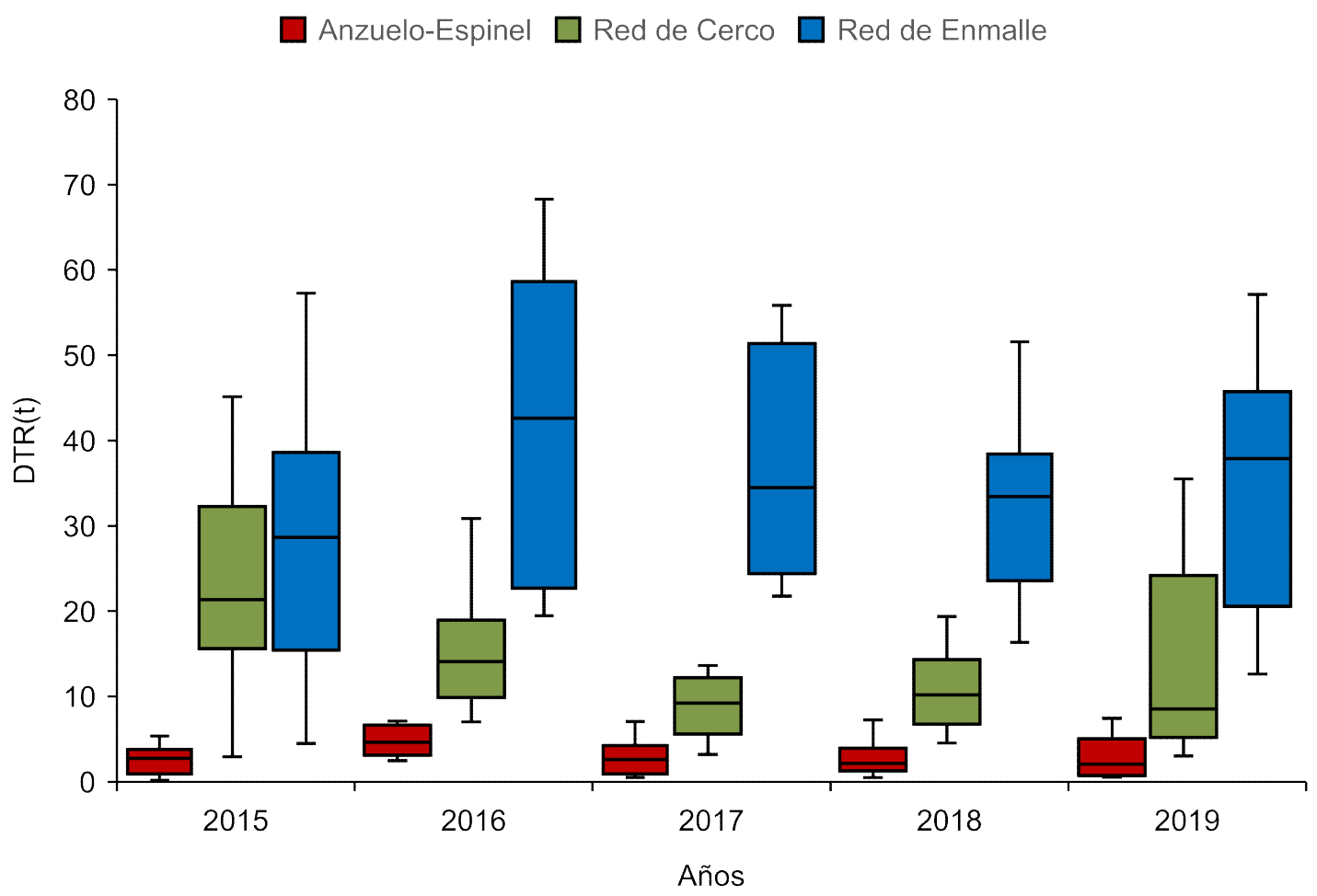

Figura 9. Variación anual del Desembarque Total Registrado [DTR(t)] por la Flota Pesquera de Yarinacocha, Ucayali (2015-2019), según las artes de pesca utilizadas
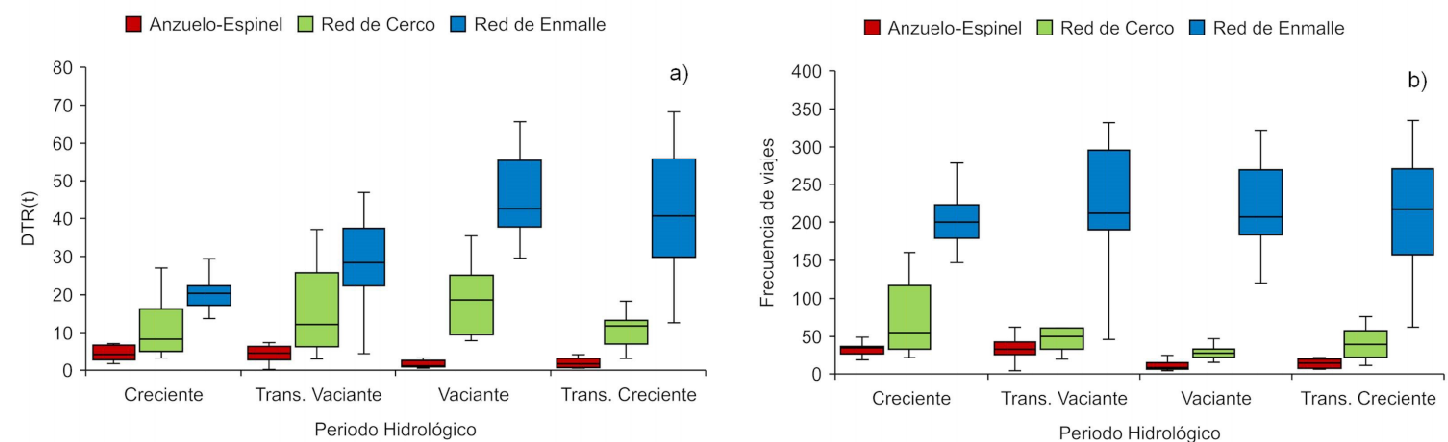

Figura 10. Desembarque Total Registrado - DTR(t) (a) y Frecuencia de viajes (b) con las principales artes de pesca por época hidrológica en el río Ucayali (periodo 20152019)

en 2015. El desembarque promedio con anzuelo-espinel fue de $3.4 \pm 2.9$ t (Figura 9).

Los más altos con respecto al desembarque para el uso de red de enmalle se presentan en la época de vaciante y transición a creciente; mientras que, la red de cerco, en «transición a vaciante»y «vaciante», asimismo para anzuelo-espinel en «transición a vaciante». En cuanto a frecuencia de viajes, se puede observar que la red de enmalle es significativamente mayor en comparación a las demás (Figura 10). 


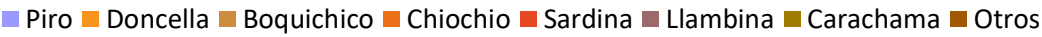

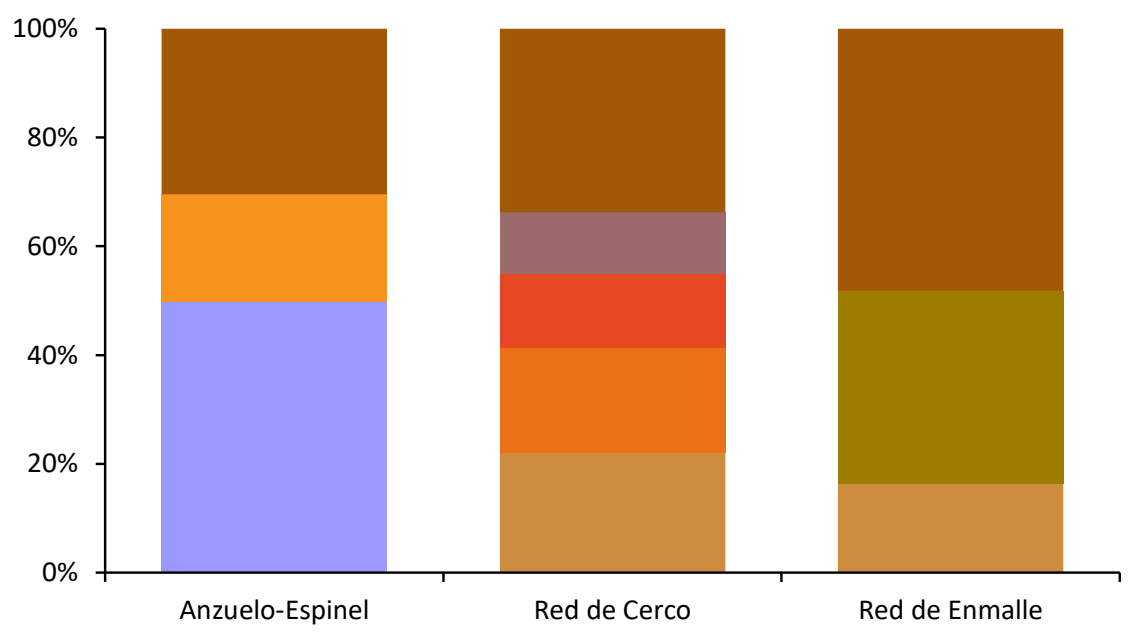

Figura 11. Aporte al Desembarque Total Registrado (DTR (\%)) de las principales especies capturadas según las artes de pesca con mayor uso en el desembarcadero de Yarinacocha, Ucayali (periodo 2015-2019)

En cuanto al desembarque de especies por arte y aparejo de pesca, se identificó que los desembarques con anzuelo estuvieron constituidos en aproximadamente $70 \%$ por «piro»y «doncella, en tanto que las especies «boquichico», «chiochio, «sardina»y «llambina» aportan en conjunto el 66\% del desembarque de la red cerco. Asimismo, «boquichico»y «carachama» fueron las principales especies capturadas por la red enmalle (Figura 11). Las especies desembarcadas en Yarinacocha se caracterizan por ser de porte medio y pequeño; no obstante, la explotación de especies de gran tamaño o de alta demanda comercial, como por ejemplo «gamitana», «paco»y «paiche» se presenta de manera intermitente o nula coincidiendo, con lo reportado por García et al., (2012) en Iquitos, Perú.

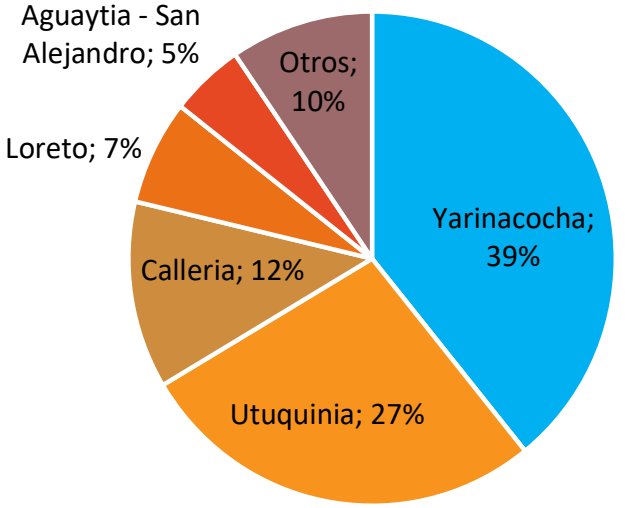

Figura 12. Variación porcentual de frecuencia de viajes en las subcuencas identificadas por la Flota Pesquera Comercial de Yarinacocha, Ucayali (2015-2019)

\section{Subcuencas}

Las subcuencas más frecuentadas por la flota pesquera fueron Yarinacochay 


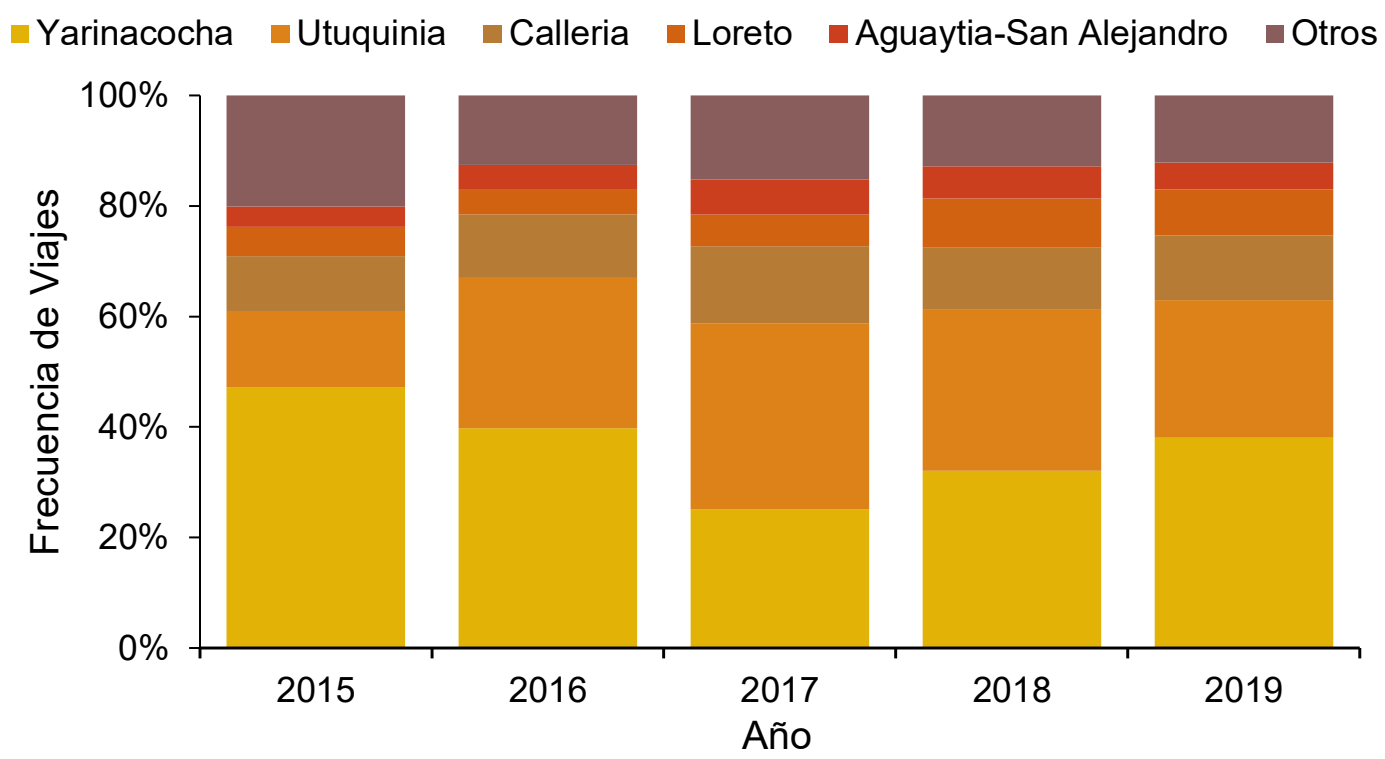

Figura 13. Variación anual de frecuencia de viajes por subcuencas de la Flota Pesquera Comercial de Yarinacocha, Ucayali (2015-2019)

Utuquinía en el periodo en estudio (Figura 12), debido a su relativa cercanía al desembarcadero y a la poca autonomía de las embarcaciones. La frecuencia de viajes a la subcuenca de Yarinacocha muestra una tendencia decreciente, desplazándose la flota pesquera hacia otras subcuencas, como Utuquinía y Callería (Figura 13).

\section{ConClusiones}

- No se presentaron diferencias significativas en los desembarques promedios anuales del periodo 2015-2019, pero hubo diferencia significativa entre meses de los periodos hidrológicos.

- Las órdenes taxonómicas que aportan mayor desembarque fueron los Siluriformes y Characiformes. Asimismo, la categoría trófica de mayor volumen de desembarque fue la omnívora, seguido de los detritívoros y piscívoros.

- Las principales especies desembarcadas fueron «carachama» y «boquichico».
- Las redes de enmalle fueron las de mayor importancia, seguidas de las redes de cerco.

- Las principales subcuencas explotadas por la flota de Yarinacocha fueron Yarinacocha y Utuquinía, lugares cercanos al desembarcadero

- Se obtuvieron bajos rendimientos e índices de Captura por Unidad de Esfuerzo (CPUE).

\section{Literatura Citada}

1. Allan J, Abell R, Hofan Z, Revenga $C$, Taylor B, Welcome $R$, Winemiller

K. 2005. Overfishing of inland waters. BioScience 55: 1041-1051. doi: 10.1641/ $0006-3568(2005) 055$ [ 1041 : OOIW]2.0.CO;2

2. Barletta M, Jaureguizar AJ, Baigun $C$, Fontoura NF, Agostinho AA, Almeida-Val VM, Val AL, et al. 2010. Fish and aquatic habitat conservation in South America: a continental overview 
with emphasis on neotropical systems. J Fish Biol 76: 2118-2176. doi: 10.1111/ j.1095-8649.2010.02684.x

3. Barthem R, Goulding M. 2007. Um ecossistema inesperado: a Amazônia revelada pela pescaBelem, Brasil: Amazon Conservation Association (ACA). 241 p.

4. Batista V, Petrere M. 2003. Caracterización de la producción comercial de pescado desembarcado en Manaus, estado de Amazonas, Brasil. Acta Amazon 33: 53-66.

5. Cadima E. 2003. Manual de evaluación de recursos pesqueros. Documento técnico de pesca. $\mathrm{N}^{\circ}$ 393. Roma, Italia: FAO. 162 p.

6. Cardoso R, Carvalho C. 2007. Desembarque e esforço de pesca da frota pesqueira comercial de Manicoré (Médio Rio Madeira), Amazonas, Brasil. Acta Amazon 37: 605-611.

7. Doria C, Ruffino M, Hijazi N, Cruz $R$. 2012. A pesca comercial na bacia do rio Madeira no estado de Rondônia, Amazônia brasileira. Acta Amazon 42: 29-40.

8. Fernandes L, Vicentini R, Batista $V$. 2009. Caracterização do uso de malhadeiras pela frota pesqueira que desembarca em Manaus e Manacapuru, Amazonas. Acta Amazon 39: 405-413.

9. García $A$, Vargas $G$, Tello $S$, Duponchelle F. 2012. Desembarque de pescado fresco en la ciudad de Iquitos, Región Loreto - Amazonía Peruana. Folia Amazónica 21: 45- 52. doi: 10.24841/ fa.v21i1-2.31

10. Hanek G. 1982. La pesquería en la Amazonía Peruana: presente y futuro. Doc. Tec. de Pesca 81. Roma, Italia: FAO. $350 \mathrm{p}$.

11. Jézéquel C, Tedesco PA, Bigorne R, Maldonado-Ocampo JA, Ortega H, Hidalgo M, Martens K, TorrenteVilara G, et al. 2020. A database of freshwater fish species of the Amazon Basin. Sci Data 7: 96. doi: 10.1038/ s41597-020-0436-4
12. Junk J, Furch K. 1985. The physical and chemical properties of Amazonian waters and their relationships with the biota. In: Prance GT, Lovejoy TE (eds). Key environments: Amazoniana. p 3-17.

13. Lopes G, Catarino M, Lima A, Freitas C. 2016. Small-scale fisheries in the Amazon basin: General patterns and diversity of fish landings in five subbasins. Bol Inst Pesca 42: 889-900. doi: 10.20950/1678-2305.2016v42n4p889

14. Lowe-McConnell R. 1975. Fish communities in tropical freshwaters: their distribution, ecology and evolution. London: Stony Brook Foundation. 337 p.

15. Ortega H, Hidalgo M, Treveiro G, Correa E, Cortijo A, Meza V, Espino J. 2012. Lista anotada de los peces de aguas continentales del Perú: estado actual del conocimiento, distribución, usos y aspectos de conservación. Lima, Perú: Ministerio del Ambiente. $58 \mathrm{p}$.

16. Riofrio J. 1998. Características de la pesquería comercial de consumo en Pucallpa (Ucayali- Perú). Rev Inv Pec 9: 67-73.

17. Tello S. 1995. Relevamiento de información sobre captura y esfuerzo pesquero con destino a ciudades. Iquitos, Perú: Instituto de Investigaciones de la Amazonia Peruana. $67 \mathrm{p}$.

18. Vela A, Zorrilla L, Vásquez A, DañinoPérez A. 2016. Análisis de los desembarques de pescado fresco en la ciudad de Pucallpa, Región Ucayali. FoliaAmazónica 22: 7. doi: 10.24841/fa.v22i1-2.27

19. Wasiw J, Riofrío J, Muñoz V. 2012. Monitoreo de la pesquería comercial en Pucallpa y Yarinacocha 2010 (Ucayali Perú). IMARPE 39: 288-293.

20. Welcomme R. 1979. Fisheries ecology of flood plain rivers. London: Longman Group Limited. $317 \mathrm{p}$.

21. Zorrilla E, Vela A, Muro P, DañinoPérez A. 2016. Características de la pesquería comercial en la cuenca del río Ucayali. Folia Amazonica 25: 159-166. 